\title{
The Importance for Health Care Providers Communicate With the Patient in their Native Language
}

\author{
Poole $\mathrm{XY} *$ \\ Duke University, USA
}

*Corresponding author: Ximena Yanez Poole, Duke University, 307 Trent Dr Durham NC 27710, USA, Tel: 9196361867; Email: ximena.yanez.poole@duke.edu

\section{Commentary}

Volume 3 Issue 6

Received Date: November 19, 2019

Published Date: November 27, 2019

DOI: $10.23880 /$ nhij-16000208

\section{Commentary}

In all my years of experience teaching Medical Spanish to nursing students and other health care workers here in the triangle area in North Carolina, I have seen how important is to communicate with patients in their native language. Patients are able to understand what is happening with their health, relatives or their children's health, diagnosis, treatments and procedures. For health care providers is easier to explain conditions of the patients, teach them about treatment options and recommendations.

Interpretation system has been a big help in the health field but patients prefer when the health care provider speaks directly to them and they are able to have eye contact with the patient. It is important to be aware about culture differences, topics such death, sexuality and women's health must be addressed with care and respect, also be careful about making jokes that might not translate well or appropriately into the patient's native language.

I always advise to my students do not be afraid to speak in Spanish, do not worry about pronunciation or using a correct grammar, it is ok make mistakes. Patients are highly grateful when the health care provider speaks in Spanish and is able to communicate directly to them, patients and relatives will feel more comfortable.

Students of Medical Spanish classes at Duke University School of Nursing have done a wonderful job in all of these years, I am very proud of them for their dedication, responsibility, eager to learn and curiosity. Having students from low to advance level of Spanish and to see them at the end of the course how much they have learned of medical vocabulary, grammar, conversational Spanish and the most important Latin American culture. One example to be aware of the use the word medication instead of "drug" when we need to explain the medication to a Latino patient, for Latinos the word drug is used for illegal drugs like cocaine, heroin, etc. when you have a Hispanic patient use the right word.

For me personally is very important also to teach them about the differences among Latino American countries, like food, celebrations, expressions and native dialects. No all Latinos eat spicy food, burritos or tortillas. We are diverse! 